\title{
Technique for Single-Graft Lateral Ankle Stabilization: A Cadaveric Biomechanical Study
}

\author{
by Craig E Clifford, DPM, MHA ${ }^{1 \rrbracket}$, Kevin M McCann, DPM, MHA ${ }^{1 凶}$
}

The Foot and Ankle Online Journal 4 (7): 3

Several surgical procedures have been described for treatment of lateral ankle instability with varying degrees of success and complications. The purpose of this study was to describe a modification to the Bröstrom procedure that allows for repair when lateral soft tissues are insufficient for primary repair. Cadaveric specimens were tested in a Telos Stress Device with lateral ligaments intact, transected, and with a single-graft repair. Talar tilt angle and anterior displacement index were measured and compared between each phase of the study. There was no significant difference between talar tilt angle of specimens with ligaments intact and ligaments grafted $(p=.2295)$. There was no significant difference between anterior displacement index of specimens with ligaments intact and ligaments grafted $(p=.6482)$. A cadaveric model is used to demonstrate that lateral ankle ligaments can be repaired using a new single-graft technique, and is comparable to presectioned intact lateral ankle ligaments.

Key words: Ankle Instability, Tendon Graft, Biotenodesis Anchors, Talar Tilt, Anterior Displacement.

Accepted: June, 2011

Published: July, 2011

This is an Open Access article distributed under the terms of the Creative Commons Attribution License. It permits unrestricted use, distribution, and reproduction in any medium, provided the original work is properly cited. (The Foot and Ankle Online Journal (www.faoj.org), 2011 All rights reserved.

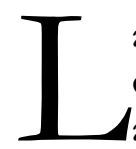
ateral ankle instability is a chronic, debilitating condition that commonly develops from an acute injury. Chronic instability has been described as a progressive process that results from the anatomic changes following an acute injury. These initial anatomic changes predispose the joint to further injury, and eventual chronic instability. ${ }^{1}$

\footnotetext{
Address correspondence to: Craig Clifford, DPM, MHA; Franciscan Foot \& Ankle Institute, Federal Way, WA

E-mail: craigclifford@,fhshealth.org

1,2 PGY2; Franciscan Foot \& Ankle Institute; $345099^{\text {th }}$ Ave S., Ste 306, Federal Way, WA 98003.
}

The Bröstrom procedure was described for primary repair of ruptured lateral ligaments. ${ }^{2}$ Modifications have been made to this procedure to reinforce the construct, including incorporation of the capsular structures into the repair for added strength. ${ }^{3}$ The modified Bröstrom procedure remains the goldstandard to which most reconstructive procedures are compared, and has been found to provide good to excellent results in $91 \%$ of patients at 26-year followup. ${ }^{4}$ Despite the favorable outcomes of the Bröstrom procedure, it is not always possible due to overattenuation of lateral soft tissues, or poor-quality of soft tissues due to previous surgery. ${ }^{5}$ 


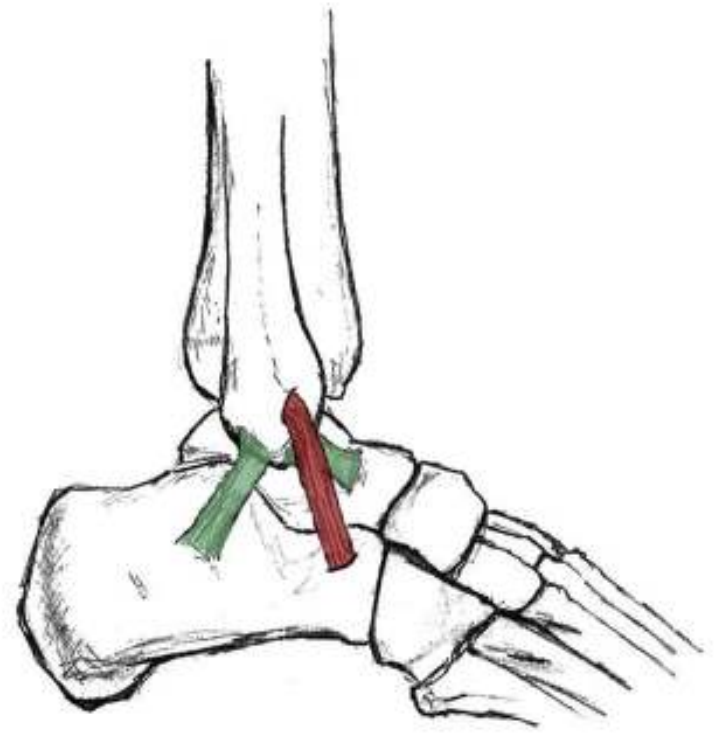

Figure 1 Single ligament graft (red) at the approximate bisection of the angle between the calcaneofibular ligament (CFL) and anterior talofibular ligament (ATFL) (green).

When primary repair of torn ligaments is not possible, many surgeons will turn to tendon transfers or free tendon grafts. More than fifty reconstructive surgical procedures have been described with varying degrees of success and complications. A common long-term complication of tendon transfers and grafting procedures is postoperative stiffness with delayed return to full function. Other reported complications are wound dehiscence, nerve damage, increased risk of degenerative changes, and recurrent instability. ${ }^{5}$

Considering these complications, it may be desirable for the surgeon to address ankle instability with a modification to the Bröstrom procedure that allows for repair even when lateral soft tissues are friable or have been previously operated upon. The purpose of this study was to test the stability of a single-graft lateral ankle ligament reconstruction technique in cadaveric specimens. The technique creates a single ligament at the approximate bisection of the angle between the calcaneofibular ligament (CFL) and the anterior talofibular ligament (ATFL) with a free tendon graft.

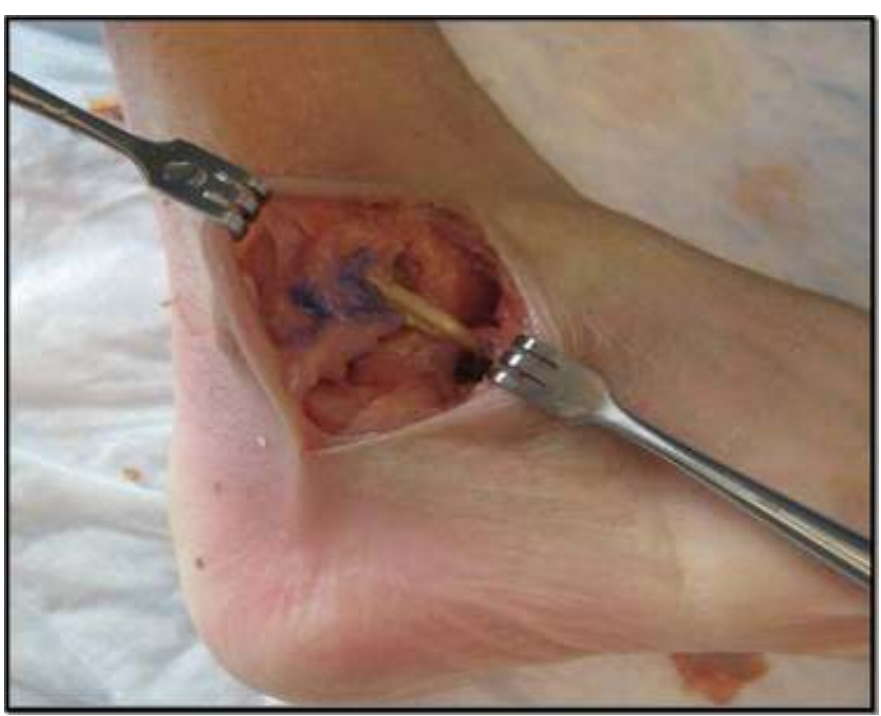

Figure 2 Completed Single free-tendon graft in a cadaver specimen.

This procedure would be indicated in patients who have had failure of the Bröstrom procedure, or when the Bröstrom procedure is not possible due unresolvable damage to lateral soft tissues or prior surgery.

\section{Methods}

Six cadaveric specimens were selected for the study, including four right lower extremities and two left lower extremities. All specimens were sectioned at the mid-tibial level. Other materials included flexor digitorum longus tendon grafts harvested from each specimen, Arthrex tenodesis anchors, a Telos Stress Device, an Orthoscan Mini C-Arm, and a radiolucent table.

Specimens were placed in the Telos Stress Device on the radiolucent table to measure talar-tilt and anterior displacement using standard technique. Ankle stress fluoroscopic images were taken with the Telos device loaded at $15 \mathrm{daN}$ of force for talar tilt, followed by $15 \mathrm{daN}$ of force for anterior displacement. This protocol was completed after each of three technique phases, as described below. 


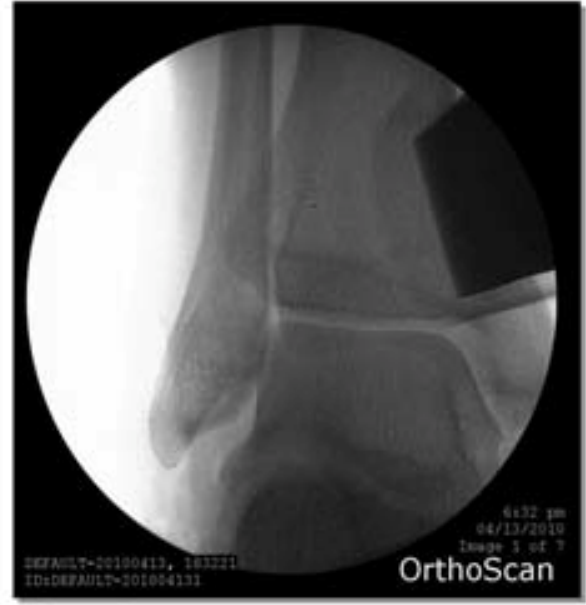

Figure 3a

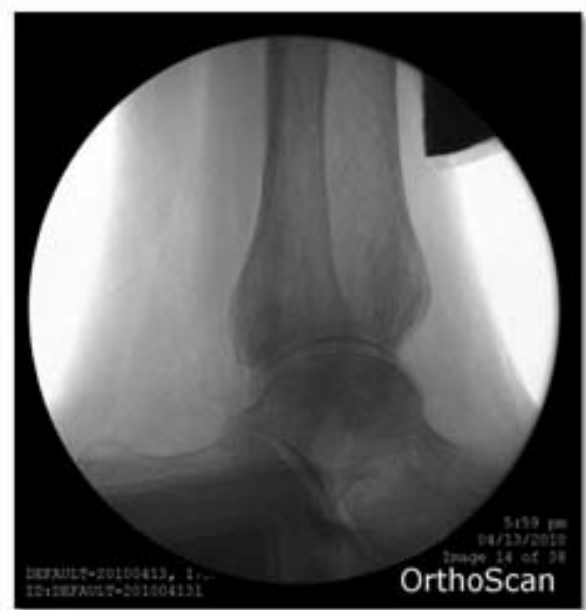

Figure 3d

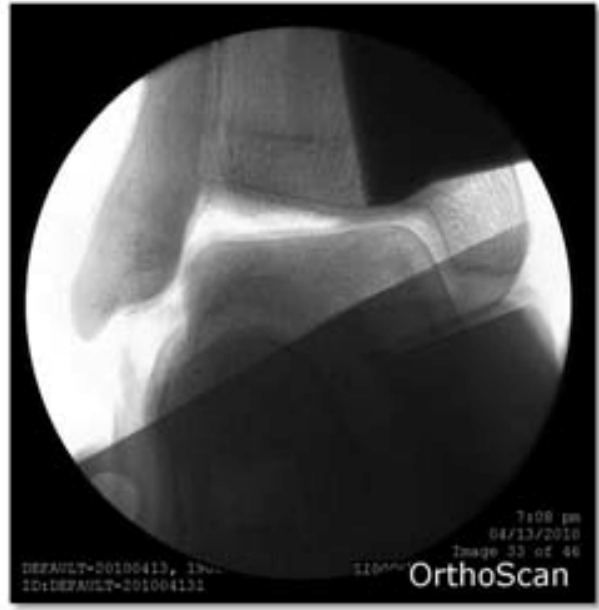

Figure 3b

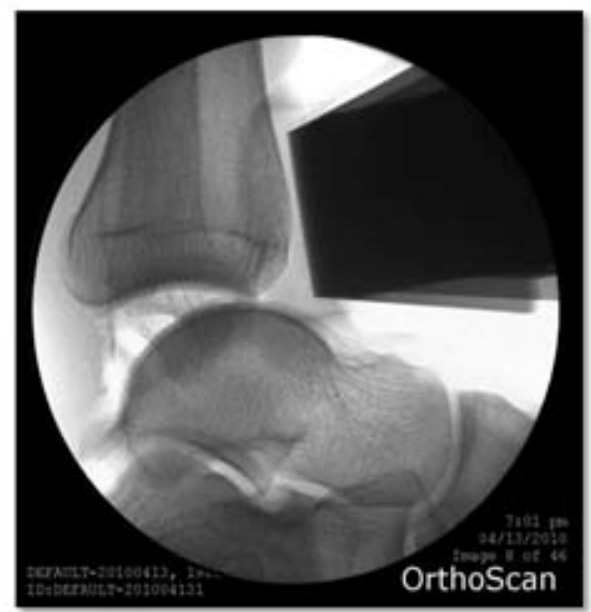

Figure 3e

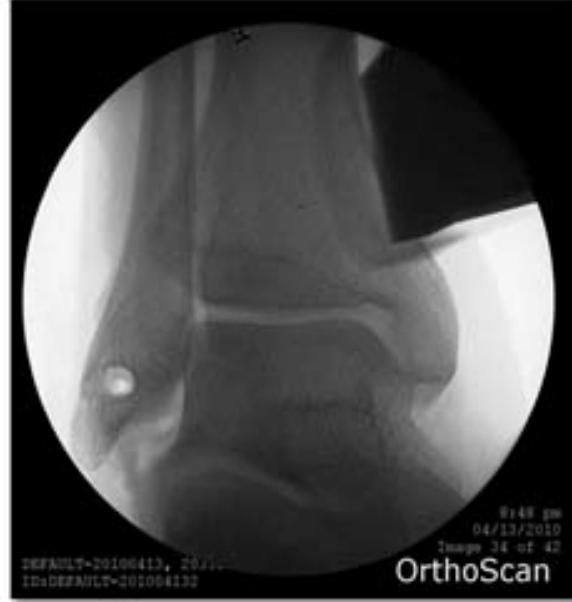

Figure 3c

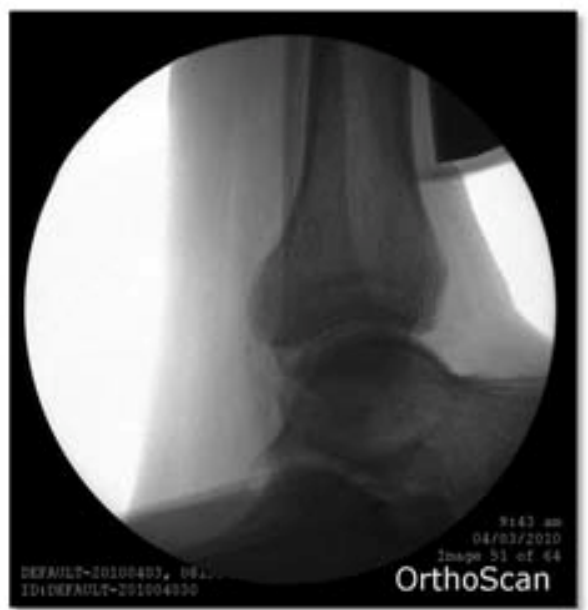

Figure 3f

Figure 3 Fluoroscopic Stress-Exam of Talar-Tilt (a) with intact lateral ligaments, (b) after sectioning ligaments, (c) after applying graft technique; and Anterior Displacement (d) with intact lateral ligaments, (e) after sectioning ligaments, (f) after applying graft technique.

\section{Phase 1: Intact Ligaments}

A curvilinear incision was made overlying the sinus tarsi. Dissection was carried down to the ankle joint capsule. The peri-capsular structures were dissected so as to identify and preserve the anterior talofibular ligament(ATFL) and calcaneofibular ligament(CFL), which were tagged with suture. The specimens were tested using the Telos Stress Test Protocol. (Fig. 1)
Phase 2: Sectioned Ligaments

The ATFL and CFL were identified and isolated at the previously identified tags, and sharply transected at their midportion. The specimens were tested using the Telos Stress'Test Protocol. 
Phase 3: Single Graft

Flexor digitorum longus tendon graft was acquired from each specimen. The distal end of the graft was attached to the anterior calcaneal process at the sinus tarsi using a $7.0 \times 23 \mathrm{~mm}$ biotenodesis anchor (Arthrex Inc. Naples, FL). The ankle joint was held at 90 degrees with the subtalar joint maximally everted. The proximal end of free tendon graft was attached $10 \mathrm{~mm}$ from the distal tip of the fibula through an anterior to posterior drill hole, using a $5.5 \times 15 \mathrm{~mm}$ biotenodesis anchor. (Fig. 2) The specimens were tested using the Telos Stress Test Protocol.

Fluoroscopic images of each limb were analyzed using ImageJ (National Institute of Health, USA) to measure talar tilt angle, and anterior displacement index. (Fig. 3) Talar tilt angle $\left(^{\circ}\right)$ was measured as the angle between the articular surfaces of talar dome and tibial plafond in the mortise radiograph. Anterior displacement index (\%) was measured by superimposing concentric circles on the talar dome and the tibial plafond in the lateral radiograph. ${ }^{6}$ The distance between the centers of the circles was measured. This distance was divided by the length of the tibial joint surface, and multiplied by 100 . The talar tilt angle and anterior displacement indeces were statistically compared using the paired $t$ test.

\section{Results}

In the six limbs on which the protocol what performed, the average talar tilt was $4.87^{\circ}$ with ligaments intact, $15.7^{\circ}$ with ligaments sectioned and $5.13^{\circ}$ with single-graft reconstruction. (Fig. 4) There was a significant difference between the talar tilt angle of specimens with ligaments sectioned and ligaments grafted $(p=.001)$. There was no significant difference between talar tilt angle of specimens with ligaments intact and ligaments grafted $(p=.2295)$. The average anterior displacement index was $17.1 \%$ with ligaments intact, $31.5 \%$ with ligaments sectioned, and $18.2 \%$ with single-graft reconstruction. (Fig. 5)

(C) The Foot and Ankle Online Journal, 2011
There was a significant difference between the anterior displacement index of specimens with ligaments sectioned and ligaments grafted $(p=.0008)$. There was no significant difference between anterior displacement index of specimens with ligaments intact and ligaments grafted $(p=.6482)$.

\section{Discussion}

The single-graft lateral ankle reconstruction differs from anatomic reconstructive procedures in that the calcaneofibular and anterior talocalcaneal ligaments are not reconstructed. Rather, the approximate average axis of these ligaments is stabilized in a single graft technique. This approach is similar to the procedure described by Evans, ${ }^{7}$ who routed the proximal portion of the peroneus brevis tendon through a drill hole in the fibula. In Evans' procedure the distal insertion of the tendon was left intact, thereby stabilizing the lateral ankle complex with a single graft at a point midway between the axis of the CFL and the ATFL. The Evans' procedure had the disadvantage of violating the peroneus brevis. The procedure also resulted in the graft crossing not only the ankle joint and subtalar joints, but by leaving the distal insertion of the brevis tendon intact, it also crossed the calcaneocuboid, and cuboid-metatarsal joints. Karlsson, et al., ${ }^{8}$ reported long-term results of the Evans' procedure, and noted that only $50 \%$ of patients reviewed had satisfactory long-term results. Poor results were related to decreased inversion range of motion, and recurrent instability, especially with anterior displacement. Karlsson, et al., concluded that the Evans' procedure deteriorates with time, and suggested that more complex reconstructions be used.

Other, non-anatomic procedures have been described with varying success. Westin, et al., described their results using a procedure of treating lateral ankle instability with extensor digitorum brevis muscle. In this procedure, the proximal attachment of the extensor digitorum brevis is transferred to the anterior border of the lateral malleolus. This resulted in a lateral ankle repair with an axis approximately midway between the CFL and ATFL axes. Westin et al. reported that the procedure is a "very good alternative" for functional instability, as it is a dynamic repair. 


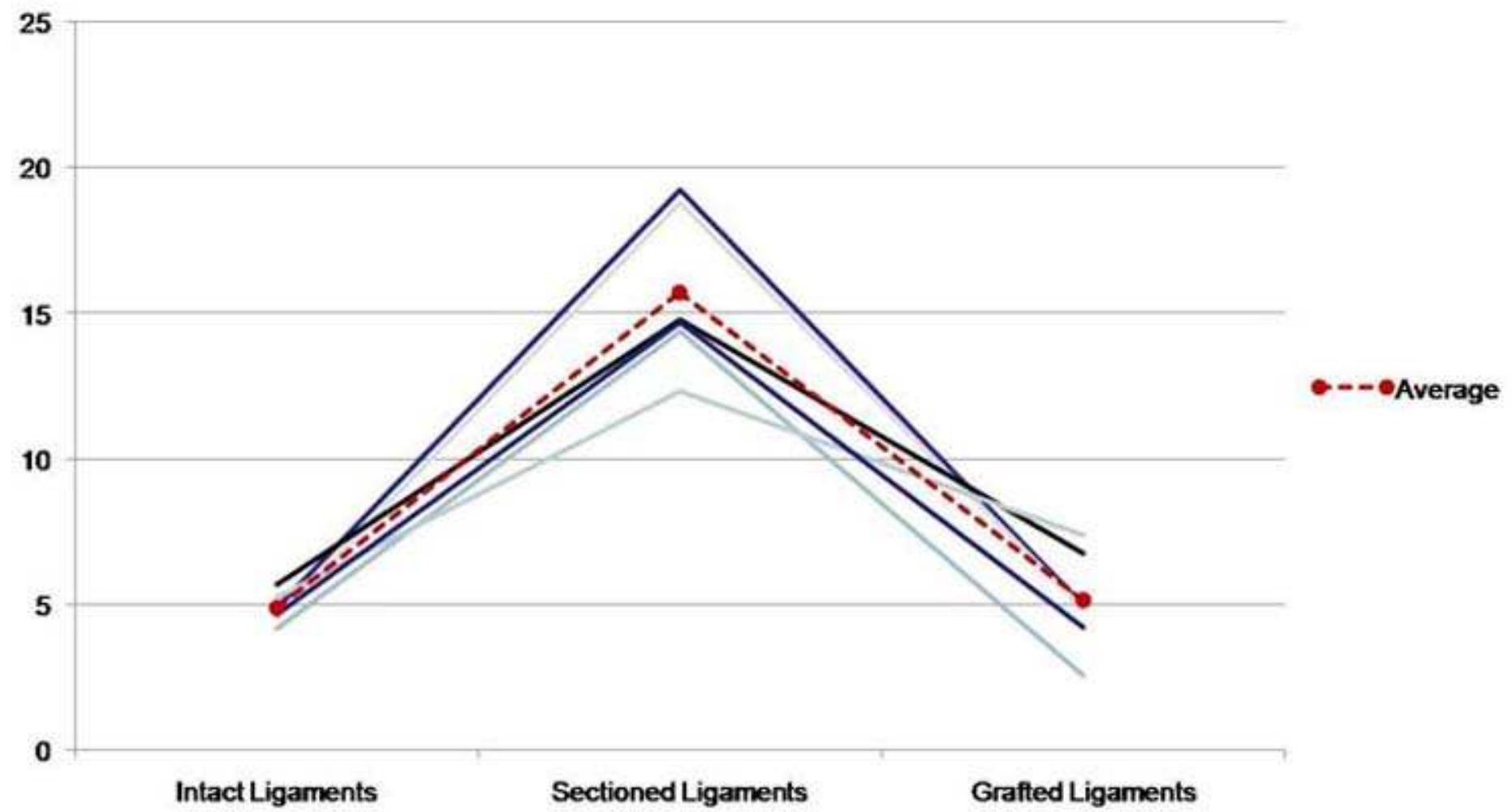

Figure 4 Results of Talar-Tilt Angle $\left(^{\circ}\right)$ measurements before sectioning, after sectioning, and after completed graft.

Jones, et al., described a minimally invasive, nonanatomic procedure using woven polyester tape as a graft material. ${ }^{10}$ In this procedure, the graft was passed between two drill holes corresponding to the distal fibula, and the base of the fifth metatarsal. In this way, the graft material created a non-anatomic reconstruction, approximately midway between the axes of the CFL and the ATFL. Jones et al. reported excellent results, with no surgical complications. The only functional complication reported was that of limited inversion on clinical examination.

Minimally invasive surgical techniques are appealing due to the theoretical reduction in complications by avoiding extensive dissection. Neurovascular injuries, however, can occur with minimal or percutaneous techniques due to limited visualization of anatomic structures. Klammer, et al., investigated the topographic anatomy of a percutaneous anatomic ankle stabilization technique, and found that neurovascular injuries are most common to the medial calcaneal branch of the tibial nerve with reconstruction of the calcaneofibular ligament. ${ }^{11}$
Several alternatives for graft fixation have been discussed in the literature, including bone anchor, interference screws, screw and washer, and simple suture. The present study uses interference screws as this fixation has been shown to be suitable for lateral ankle ligament augmentation. Jeys et al. compared Mitek GII anchors to Arthrex Biotenodesis screws, and found that the Arthrex screws had greater strength to failure, and less elongation at the point of failure. $^{12}$

\section{Conclusion}

The results obtained during the course of this study suggest that lateral ankle ligaments can be successfully repaired using a new single-graft technique in a cadaveric model. The stability of the single-graft ankle stabilization construct is comparable to that of intact lateral ankle ligaments. The single-graft lateral ankle reconstruction differs from anatomic reconstructive procedures in that the calcaneofibular and anterior talocalcaneal ligaments are not reconstructed. 


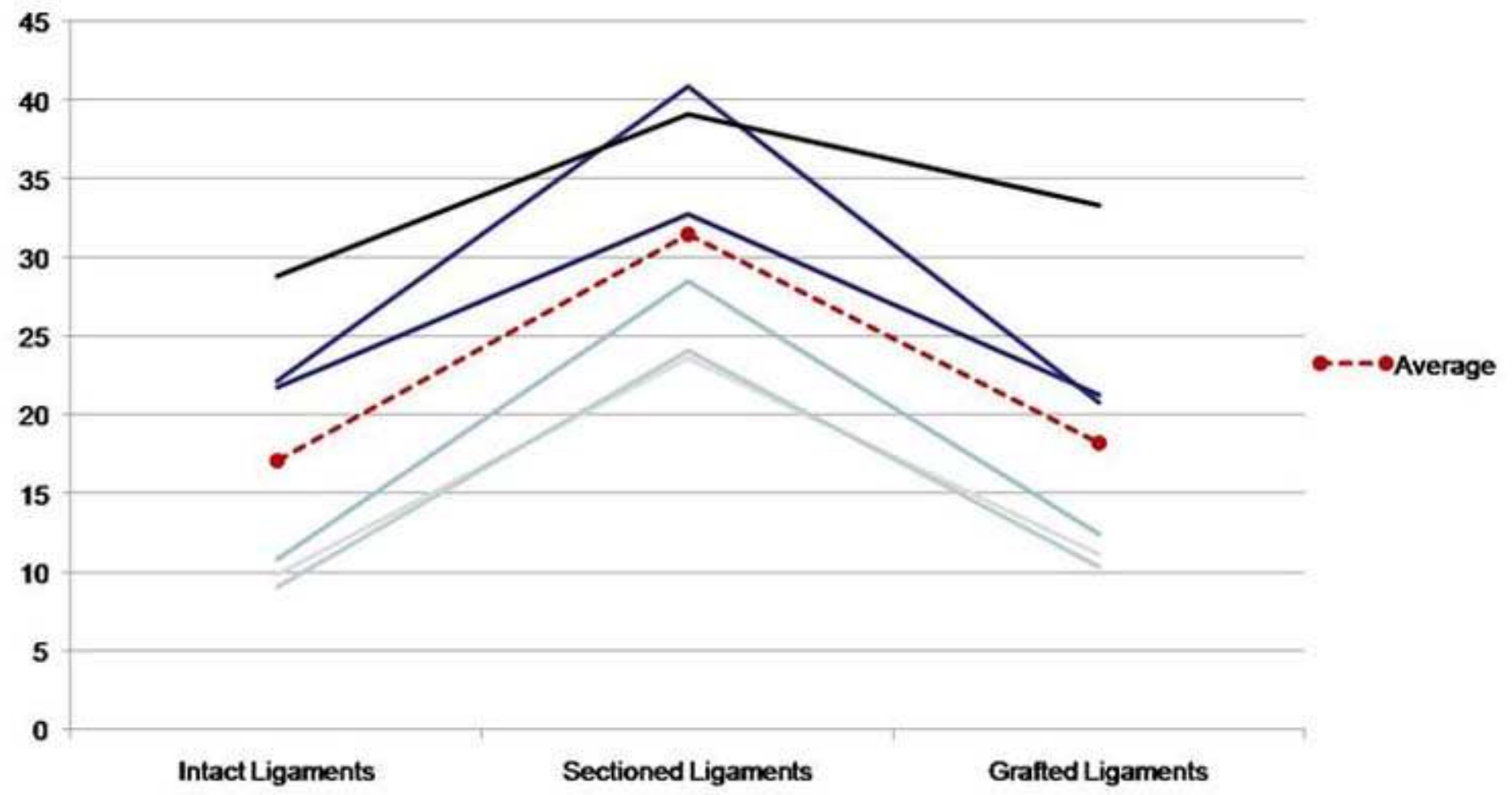

Figure 5 Results of Anterior Displacement Index (\%) before sectioning, after sectioning, and after completed graft.

Rather, the approximate average axes of these ligaments are stabilized in a single graft technique.

The advantage of this procedure is that it recreates the stability of both the ATFL and the CFL, using a single-graft technique that is less invasive and requires less allograft material than previously described ligament reconstructions. Future work is needed to compare the single-graft technique anatomic reconstructive techniques. In vivo studies with long term follow up are also needed to determine dynamic stability and sequelae.

\section{References}

1. Hertel J. Functional anatomy, pathomechanics, and pathophysiology of lateral ankle instability. J Athletic Training 2002 37: 364-375.

2. Brostrom L. Sprained ankles: Clinical observations in recent ligament ruptures. Acta Chir Scand 1965 132: 537-550.

3. Paden MH, Stone PA, McGarry JJ. Modified Brostrom lateral ankle stabilization utilizing an implantable anchoring system. J Foot Ankle Surg 1994 33: 617-622.

4. Bell SJ, Mologne TS, Sitler DF, Cox JS. Twenty-six-year results after Brostrom procedure for chronic lateral ankle instability. Am J Sports Med 2006 34: 975-878.

5. Sammarco VJ. Complications of lateral ankle ligament reconstruction. Clin Orthop Relat Res 2001 391:123-132.

6. Lindstrand A, Mortensson W. Anterior instability in the ankle joint following acute lateral sprain. Acta Radiologica Diagnosis 197718: 529-539.

7. Evans DL. Recurrent instability of the ankle joint: A method of surgical treatment. J R Soc Med 1953 46: 343-344. 
8. Karlsson J, Bergsten T, Lansinger O, Peterson L. Lateral instability of the ankle treated by the Evans procedure: A longterm clinical and radiological follow-up. JBJS 198870B: 476-480. 9. Westlin NE, Vogler HW, Albertsson MP, Arvidsson T, Montgonery F. Treatment of lateral ankle instability with transfer of the extensor digitorum brevis muscle. J Foot Ankle Surg 2003 42:183-192.

10. Jones A, Sidhom S, Sefton G. A minimally invasive surgical technique for augmented reconstruction of the lateral ankle ligaments with woven polyester tape. J Foot \& Ankle Surg 2007 46: 416-423.

11. Klammer G, Schlewitz G, Stauffer C, Vich M, Espinosa N. Percutaneous lateral ankle stabilization: An anatomic investigation. Foot Ankle Int 2011 32: 66-70.

12. Jeys L, Korrosis S, Stewart T, Harris NJ. Bone anchors or interference screws?: A biomechanical evaluation for autograft ankle stabilization. Am J Sports Med 2004 32: 1651-1659. 